\title{
The Research on the Correlation of Thyroid Function and Serum Immunological Indexes in Patients with Hashimoto's Thyroiditis
}

\author{
Rongqin Dai ${ }^{1}$, Yuzhi Liu ${ }^{1}$, Jinbiao Zhang ${ }^{2}$, \\ ${ }^{1}$ Institute of Thyroid Disease Affiliated to Cangzhou Medical College, Cangzhou, China \\ ${ }^{2}$ Laboratory Diagnostics Division, Cangzhou Hospital of Integrated Traditional Chinese and Western Medicine, Cangzhou, China
}

Email address:

zjb7829325@163.com (Jinbiao Zhang)

${ }^{*}$ Corresponding author

To cite this article:

Rongqin Dai, Yuzhi Liu, Jinbiao Zhang. The Research on the Correlation of Thyroid Function and Serum Immunological Indexes in Patients with Hashimoto's Thyroiditis. American Journal of Life Sciences. Vol. 8, No. 2, 2020, pp. 19-22. doi: 10.11648/j.ajls.20200802.11

Received: May 27, 2020; Accepted: July 10, 2020; Published: July 22, 2020

\begin{abstract}
Objective: To assess the correlation of thyroid function and serum immunological indexes in patients with Hashimoto's thyroiditis. Method: To select 172 patients who have been clinically confirmed with HT. And they were divided into Hashimoto hyperthyroidism group within 39 cases, Hashimoto hypothyroidism group within 92 cases and normal Hashimoto's thyroid function group within 41 cases on the basis of Thyroid function. Besides, 50 healthy controls were control group. ECLIA was adopted to test the Thyroid function and antibody indicators of every group, and ELISA was employed to detect Th1 type cytokines (IL-2, IFN- $\gamma$ ) and Th2 type cytokines (IL-6, IL-10). Result: Every group with Hashimoto's thyroiditis was evidently higher than the normal one as well as the Hashimoto hypothyroidism group was higher than the Hashimoto hyperthyroidism group and the normal one in TPOAb and TGAb levels, in which the difference has statistical significance $(\mathrm{P}<0.05)$. However, the difference between Hashimoto hyperthyroidism group and Hashimoto hypothyroidism group has no statistical significance ( $\mathrm{P}>0.05)$; Moreover, the IL-2, IFN- $\gamma$ levels of the Hashimoto hyperthyroidism group and the normal Hashimoto's thyroid function group were distinctly higher than that of the Hashimoto hypothyroidism group; And the IL-6 and IL-10 levels were obviously higher than the Hashimoto hyperthyroidism group as well as the normal Hashimoto's thyroid function group $(\mathrm{P}<0.05)$. Besides, the TSH was positively correlated with the TPOAb and the IL-10. And FT4 was negatively correlated with the TPOAb, TGAb, IL-6. Not only the FT3 and TGAb but also the TT4 and IL-6 were in negative correlation. But the other indexes have no correlation. Conclusion: The patients with Hashimoto's thyroiditis have high TPOAb and TGAb levels. In addition, the TPOAb, TGAb and cytokines levels in the serum of HT patients with different thyroid function have evidently discrepancy, which may be closely related to the occurrence and development of the disease.
\end{abstract}

Keywords: Hashimoto's Thyroiditis, Thyroid Function, Antibody, Cytokine

\section{Introduction}

The Hashimoto's thyroiditis (HT) is a typical thyroid disease about witch organ-specific and autoimmunity are mediated by T cells. The patients' thyroid autoantibodies and lymphocytes are infiltrated in thyroid tissue, which causes thyroid follicles' destroying and atrophie thyroid tissues [1]. Thyroid function can vary in patients. In the early stages of the disease, the patients' thyroid function is normal or they will emerge transient hyperfunction. With the destroying of the thyroid follicles, they will gradually manifests as hypothyroidism [2]. The causes of the HT are closely related to the body's disorder cellular immune and humoral immune function of the thyroid epithelial cells [3]. According to the research, most of the HT patients' thyroid peroxidase antibodies (TPOAb) and thyroid globulin antibodies (TGAb) titers rose [4]. At the same time, there is an imbalance of Th1/Th2 cells proportion [5]. At present, there are rare researches on the indexes expression of serum antibodies and cytokines or others of patients with different thyroid function. Therefore, by analyzing the changes in serum immune indexes of the HT patients with different thyroid function, this article is aimed at further discussing the HT pathogenesis as well as providing theory bases for the HT diagnoses and treatment. 


\section{Methods}

\subsection{General Information}

172 patients who were clinically diagnosed with HT in the endocrinology department of Cangzhou Hospital of Integrated Traditional Chinese and Western Medicine, Hebei province from November, 2017 to October, 2019 were chosen to be study objects, and they were all primary-care patients, among which there were 35 men cases, 137 women cases with age from $18 \sim 70$, and the average age were (44.07 \pm 17.83$)$ years old. They were divided into Hashimoto hyperthyroidism group within 39 cases, Hashimoto hypothyroidism group within 92 cases and normal Hashimoto's thyroid function group within 41 cases on the basis of Thyroid function. Besides, 50 healthy controls were control group, in which there were 10 men cases and 40 women cases with age from $19 \sim 70$, and the average age were $(44.33 \pm 16.57)$ years old. The general clinical data such as gender and age were compared among groups. And the differences have no statistical significance. This research has been approved by the ethics committee and all the subjects were informed and agreed.

\subsection{Diagnostic Criteria}

The HT diagnosis refers to the diagnostic criteria [6] in the 8th version of Internal Medicine published by the People's medical publishing house eighth printing Medicine, including: (1) the one with typical clinical features, such as diffusing goiter, tough texture, especially with thickening of isthmus and pyramidal lobe, etc., or TPOAb or TGAb positive, can be diagnosed; (2) the one without goiter, but with distinct TPOAb or TGAb increase as well as hypothyroidism can also be diagnosed.

\subsection{Exclusion Criteria}

(1) With the history of pituitary or hypothalamus disease; (2) The pregnant or breastfeeding woman; (3) Take medicine which affects thyroid hormone; (4) Patient with autoimmune diseases; (5) Patient with abnormal liver and kidney function, and/or cardiac insufficiency.

\subsection{Experimental Method}

\subsubsection{Specimen Collection}

Take $4 \mathrm{ml}$ fasting venous blood in the early morning for all the patients and the healthy for check-up, separate the serum timely, and place it in cryogenic refrigerator with the temperature as $-40^{\circ} \mathrm{C}$ for storage.

\subsubsection{Thyroid Function and Antibody Test}

Table 1. Comparison of serum immunological indexes in each group $(\bar{x} \pm s)$.

\begin{tabular}{|c|c|c|c|c|c|c|}
\hline Groups & TPOAb IU/ml & TGAb IU/ml & IL-2 pg/ml & IFN- $\gamma \mathrm{pg} / \mathrm{ml}$ & IL-6 pg/ml & IL-10 pg/ml \\
\hline Hashimoto hyperthyroidism group & $221.27 \pm 142.34$ & $547.53 \pm 112.13$ & $3.87 \pm 2.95$ & $6.86 \pm 3.92$ & $1.35 \pm 1.27$ & $1.48 \pm 1.42$ \\
\hline Hashimoto hypothyroidism group & $386.83 \pm 187.15$ & $878.74 \pm 152.43$ & $2.47 \pm 1.85$ & $4.09 \pm 2.65$ & $1.74 \pm 1.35$ & $2.83 \pm 2.01$ \\
\hline Normal Hashimoto's thyroid function group & $183.61 \pm 87.84$ & $501.47 \pm 116.38$ & $3.61 \pm 2.84$ & $5.54 \pm 3.10$ & $1.25 \pm 1.23$ & $1.73 \pm 1.52$ \\
\hline Normal Control Group & $23.14 \pm 13.68$ & $60.27 \pm 34.64$ & $2.08 \pm 0.93$ & $3.25 \pm 2.12$ & $1.31 \pm 1.06$ & $2.63 \pm 1.94$ \\
\hline $\mathrm{F}$ & 16.89 & 14.37 & 6.32 & 7.16 & 6.87 & 8.04 \\
\hline $\mathrm{P}$ & 0.002 & 0.012 & 0.043 & 0.037 & 0.040 & 0.036 \\
\hline
\end{tabular}

Adopt Cobas E601 electrogenerated chemiluminescence analyzer of Roche supporting assay to test thyroid function and antibody indicator, including TSH, TT4, FT4, TT3, FT3, TPOAb and TGAb.

\subsubsection{Cytokines Test}

Adopt ELISA double antibody sandwich method to test Th1 type cytokines (IL-2, IFN- $\gamma$ ) and Th2 cytokines (IL-6, IL-10), with the assays purchased from MultiSciences (Lianke) Biotech Co., Ltd. and the operation conducted strictly in accordance with the instruction manual.

\subsection{Statistic Method}

The data adopts SPSS24.0 software for analysis and processing, the metering data is expressed by $(\bar{x} \pm s)$, the comparison among multiple groups adopts analysis of variance, pairwise comparison adopts SNK-q test, and correlation analysis adopts linear correlation analysis.

\section{Results}

\subsection{Comparison of Immunological Indexes of Each Group of Serum}

TPOAb and TGAb of each HT group is distinctly higher than that of normal control group $(\mathrm{P}<0.05), \mathrm{TPOAb}$ and TGAb of each HT hypothyroidism is higher than that of HT hyperthyroidism group and normal HT thyroid function group, and the difference has statistic meaning $(\mathrm{P}<0.05)$; the difference in TPOAb and TGAb level between HT hyperthyroidism and normal HT thyroid function group has no statistic meaning $(\mathrm{P}>0.05)$; IL-2 and IFN- $\gamma$ level of HT hyperthyroidism group and normal HT thyroid function group is distinctly higher than that of HT hypothyroidism group and normal control group $(\mathrm{P}<0.05)$, IFN- $\gamma$ level of HT hypothyroidism group is higher than that of normal control group, and the difference has statistic meaning $(\mathrm{P}<0.05)$; the IL-2 level is higher than that of normal control group, but the difference has no statistic meaning $(\mathrm{P}>0.05)$; IL-6 and IL-10 level of HT hypothyroidism group is distinctly higher than that of HT hyperthyroidism group and normal HT thyroid function group $(\mathrm{P}<0.05)$, and the comparison difference of IL-6 level of HT hyperthyroidism group and normal HT thyroid function group with the normal control group has no statistic meaning $(\mathrm{P}>0.05)$; IL-10 level is lower than that of normal control group, and difference has statistic meaning $(\mathrm{P}<0.05)$. (Table 1). 


\subsection{The Correlation of Ht Thyroiditis Patients' Thyroid Function Index and Serum Immunological Index}

TSH is in positive correlation with TPOAb and IL-10, FT4 is in negative correlation with TPOAb, TGAb and IL-6, TT4 is in negative correlation with IL-6, FT3 is in negative correlation with TGAb, and other indexes has no distinct correlation among each other. (Table 2).

Table 2. The correlation of HT thyroiditis patients' thyroid function index and serum immunological index.

\begin{tabular}{llllll}
\hline \multirow{2}{*}{ thyroid function index } & \multicolumn{1}{l}{ r } & & & \\
\cline { 2 - 6 } & TPOAb & TGAb & IL-2 & IFN- $\gamma$ & IL-6 \\
\hline TSH & $0.612^{*}$ & 0.312 & -0.371 & -0.198 & 0.361 \\
FT4 & $-0.423^{*}$ & $-0.469^{*}$ & 0.215 & 0.174 & $-0.721 *$ \\
TT4 & -0.165 & -0.532 & 0.087 & 0.162 & $-0.374 *$ \\
FT3 & -0.231 & $-0.358^{*}$ & 0.145 & 0.247 & -0.293 \\
TT3 & -0.098 & -0.267 & 0.276 & 0.084 & -0.267 \\
\hline
\end{tabular}

Note: $* \mathrm{P}<0.05$.

\section{Discussions}

Thyroid peroxidase is a low glycosylated membrane-bound enzyme, and can catalyze the oxidation of iodine and the iodination of tyrosine residue of thyroglobulin. Thyroglobulin is an iodized glycoprotein existing in thyroid gland as well as a storage form of iodine in thyroid gland, and can generate thyroxine and triiodothyronine after hydrolysis [7]. TPOAb and TGAb is important symbols of HT thyroiditis. More than $80 \%$ patients have continuous TPOAb and TGAb level rise, which is the one of the important bases [8] for HT diagnosis. The combination of TPOAb and TPO reduces the synthesis [9] of thyroid hormone by containing the enzymatic activity of TPO on one hand, and destroys thyroid follicular membrane on the other hand by activating cell-mediated cytotoxic effect which the complement, and the antibody relies on, which cause the death [10] of thyroid cells in return. TGAb has enzymatic activity and can catalyze the hydrolysis of TG, and causes the TG reduction in the blood circulation and thyroid gland. The reduction of TG will certainly affect the synthesis of T3 and T4 [11]. As for whether TGAb will destroy thyroid follicle, there are different opinions. Some researches regard that TGAb can't anchor the complement and cross linking is impossible due to big epitope interval, therefore, it will not destroy the thyroid cells [12]; other researches regard that TGAb is the same as TPOAb which can destroy thyroid cells through complement anchorage and cytotoxic effect [13], thus it is to be further verified as for whether it can mediate ADCC in body. By observing HT patients' TPOAb and TGAb level of different thyroid function, it is discovered that TPOAb and TGAb of HT hypothyroidism group is distinctly higher than that of HT hyperthyroidism group and normal HT thyroid function group, while the concentration difference of TPOAb and TGAb between HT hyperthyroidism group and normal HT thyroid function group has no statistic meaning. Considering the attack process of the disease, usually HT patients with hyperthyroidism and normal thyroid function are at the early stage of the disease, the antibody level in the patient is relatively low, which has relatively small influence on the destruction of thyroid gland and the synthesis of thyroid hormone, while HT patients with hypothyroidism are usually at the end stage of the disease, the patients have relatively high antibody level in it, which will cause the reduction of thyroid hormone synthesis and the rise of TSH on one hand, which is manifested as hypothyroidism, and deteriorate the hypothyroidism on the other hand by destroying thyroid follicle and cause the injury of thyroid tissue. The correlation research between thyroid function index and TPOAb as well as TGAb indicates that TPOAb is in positive correlation with TSH and negative correlation with FT4, and has no distinct correlation with FT3, TT3 and TT4; TGAb is in negative correlation with FT3 and FT4, and has no distinct correlation with TSH, TT3 and TT4; the hypothyroidism patients have TSH rise, and FT3, FT4 reduction, which corresponds to HT hypothyroidism patients' relatively high TPOAb and TGAb level.

T cell plays an important role in the pathogenesis of HT. Many researches indicate that Th1/Th2 cytokines participate in the disease attack and development of HT [14-16]. Th1 type cytokines mainly include IL- 2 , IFN- $\gamma$, TNF- $\beta$, etc. Mediated Cellular immunity, macrophage activation, differentiation and activation of cytotoxic $\mathrm{T}$ cell are mainly secreted by Th1 cell. Th2 type cytokines mainly include IL-4, IL-6, IL-10, etc., which can promote the activation of $\mathrm{B}$ cell and generate antibody. Mediated humoral immunity response is mainly secreted by Th2 cell [17]. The balance between Th1/Th2 cytokines plays an important role in maintaining the stability of immune function of the body, its imbalance may cause the disease of immunity mediation. Many researches regards that HT patients' Th1/Th2 cell balance deviates toward Th1, some researches regard that the HT patients' cytokines imbalance deviates toward $\mathrm{Th} 2$ [5]. The research reflects that Th1 cytokines of HT hyperthyroidism group and normal HT thyroid function are distinctly higher than that of hypothyroidism group, while $\mathrm{Th} 2$ type cytokines are distinctly lower than that of hypothyroidism group. Considering the different stage of the disease, the difference between Th1/Th2 balance may play an important role in the generation and development of HT. At the early stage of HT, Th1 cell diffuses in thyroid tissue in large amount, which causes the injury of thyroid tissue directly due to the activation of cytotoxic $\mathrm{T}$ lymphocytes and macrophages and makes the thyroid hormone stored in thyroid follicle cells released into the blood in large amount. As a result, transient hyperthyroidism is 
caused. The research reflects that at the early stage of autoimmune thyroiditis attack, the expression level of IFN- $\gamma$ has increased before the rise of TGAb titer, which promotes the generation and development of autoimmune diseases [18]. IFN- $\gamma$, IL- 6 and other inflammatory cytokines can contain the expression of sodium-iodide co-conveyor in thyroid follicle cells and the synthesis of protein, which will affect the uptake activity of iodine and thyroid hormone in return and cause the abnormality of TPO activity and the abnormal release of TG in blood [19]. Under the action that Th2 cytokines promote the antibody generation, the patients' TPOAb and TGAb level rises continuously, which further cause the damage of thyroid cells and thyroid hormone, and cause hypothyroidism in return. The research on the correlation between thyroid function index and cytokines indicates that IL-10 is in positive correlation with TSH, IL-6 is in negative correlation with FT4 and TT3, which explains that the cytokines have close relation with thyroid hormone synthesis and release.

\section{Conclusions}

The relationship between thyroid function and immunological indexes in serum was observed in 172 patients with HT. The results show that, the levels of TPOAb and $\mathrm{TGAb}$ were correlated with thyroid function, high levels of TPOAb and TGAb can lead to hypothyroidism; There was Th1/Th2 imbalance in HT patients, Th1 cytokines were mainly secreted in hyperthyroidism stage, and Th2 cytokines were mainly secreted in hypothyroidism stage. TPOAb, TGAb levels and Th1 / Th2 imbalance participate in the occurrence and development of HT.

\section{Acknowledgements}

This study was supported by a project grant from: science and technology research and development guidance plan project in cangzhou (Grant No. 172302039).

\section{References}

[1] Chen Yumin, Hu Fengqiu, Huang Hui, et al. The effect of Treg and Th17 cells in Hashimoto's thyroiditis patients with normal thyroid function [J]. Medical Journal of West China, 2018, 30 (10): 1438-1442.

[2] GABALEC F, SRBOVA L, NOVA M, et al. Impact of Hashimoto's thyroiditis, TSH levels, and anti-thyroid antibody positivity on differentiated thyroid carcinoma incidence $[\mathrm{J}]$. Endokrynol Pol, 2016, 67 (1): 48-53.

[3] Chong Xianjin, Yu Qingyuan, Aman Guli·Suli Tang, et al. The effect of dexamethasone combined with thyroxine in the treatment of autoimmune thyroiditis and its effect on the related serum cytokines and $\mathrm{Th} 1 / \mathrm{Th} 2$ related cytokines [J]. Chinese Journal of Difficult and Complicated Cases, 2018, 17 (11): 1233-1237.

[4] Ma Ling, Chen Yuqiong, Du Juan, et al. Correlations between thyroid autoantibodies and thyroid function in patients with newly-diagnosed Hashimoto's thyroiditis [J]. China Journal of
Modern Medicine, 2018, 28 (17): 94-97.

[5] Ma Shifen, Wang Juanhong, Wei Wei, et al. Expression and its clinical significance of IL-4 and IL-6 in Hashimoto's thyroiditis [J]. Journal of Shanxi Medical University, 2018, 49 (12): 1483-1487.

[6] Ge Junbo, Xu Yongjian. Internal Medicine [M]. 8th Edition. Beijing: People's Medical Publishing House, 2013: 698.

[7] Liu Guanchang, Ma Shaoning. Biochemical examination [M]. 4th Edition. Beijing: People's Medical Publishing House, 2015: 316.

[8] Jin Baosheng, Fan Wei, Qi Jinyou. Effect of Elevated Serum $\mathrm{TPOAb}$ and $\mathrm{TgAb}$ on thyroid disease progression $[\mathrm{J}]$. International Journal of Laboratory Medicine, 2014, 35 (24): 3433-3436.

[9] Song YH, Li Y, Maclaren NK. The nature of autoantigens targeted in autoimmune endocrine diseases [J]. Immunology Today. 1996, 17 (5): 232-238.

[10] Liang Xiuzhen, Wang Jing, Yang Xiaoqiong, et al. Diagnostic value of thyroid autoantibodies in Hashimoto's thyroiditis: a Meta analysis [J]. International Journal of Laboratory Medicine, 2018, 39 (10): 1206-1210.

[11] Du Juan. The Relationship between Hashimoto'S thyroiditis and TCM syndrome type And association between thyroid autoantibodies and thyroid function in patients [D]. [Master's thesis]. Nanjing: Nanjing University of Chinese Medicine, 2017: 3.

[12] Xiao Fang. Study on potential roles of NETs in the pathogenesis of Hashimoto's thyroiditis and underlying mechanisms [D]. [Doctoral dissertation]. Jinan: Shandong University, 2018: 34.

[13] Zhao Tingting. Correlation between Hashimoto'S thyroiditis with normal thyroid function and insulin resistance in patients with thyroid nodules [D]. [Master's thesis]. Dalian: Dalian Medical University, 2018: 9.

[14] Jiang Yanping, Zhang Huili. Preliminary study of Th1 / Th2 Imbalance in Different Types of Autojmmune Thyroid Disease [J]. Journal of Medical Research, 2014, 43 (6): 111-114.

[15] Guo Y, Zynat J, Xing S, et al. Immunological changes of T helper cells in flow cytometersorted CD4 $(+) \mathrm{T}$ cells from patients with Hashimoto's thyroiditis [J]. Exp Ther Med, 2018, 15 (4): 3596-3602.

[16] Mikos H, Mikos M, Obaramoszyńska M, et al. The role of the immune system and cytokines involved in the pathogenesis of autoimmune thyroid disease (AITD) [J]. Endokrynol Pol, 2014, 65 (2): 150-155.

[17] Inoue $N$, Watanabe $M$, Nakaguchi $A$, et al. Functional polymorphisms affecting Th1 differentiation are associated with the severity of autoimmune thyroid diseases [J]. Endocrine Journal, 2017, 64 (7): 695-703.

[18] Yu S, Sharp GC, Braley-Mullen H. Thyrocytes responding to IFN-gamma are essent ial for development of lymphocytic spontaneous autoimmune thyroiditi $\mathrm{s}$ and inhibition of thyrocyte hyperplasia [J]. J Immunol, 2006, 176 (2): 1259-1265.

[19] Wang Lele, Chen Xiaopan. A study of MiRNA in autoimmune thyroid disease [J]. Journal of Hainan Medical University, 2018, 24 (21): 1939-1942. 